\title{
On the significance of asperity models predictions of rough contact with respect to recent alternative theories
}

\author{
M.Ciavarella
}

July 30, 2021

\begin{abstract}
Recently, it has been shown that while asperity models show correctly qualitative features of rough contact problems (linearity in area-load, negative exponential dependence of load on separation which means also linearity of stiffness with load), the exact value of the coefficients are not precise for the idealized case of Gaussian distribution of heigths. This is due to the intrinsic simplifications, neglecting asperity coalescence and interaction effects. However, the issue of Gaussianity has not been proved or experimentally verified in many cases, and here we show that, for example, assuming a Weibull distribution of asperity heigths, the area-load linear coefficient is not much affected, while the relationships load-separation and therefore also stiffness-load do change largely, particularly when considering bounded distributions of asperity heigths.

It is suggested that Gaussianity of surfaces should be further tested in experiments, before applying the most sophisticated rough contact models based on the Gaussian assumption.
\end{abstract}

Address: Politecnico di BARI. V.le Gentile 182, 70125 Bari-Italy.

Email mciava@poliba.it, tel +390805962811 fax +390805962777

\section{Introduction}

The GW model (Greenwood \& Williamson, 1966) has in the last 15-20 years been questioned and Persson's theories (Persson, 2001, 2007) have been developed which, with significantly more elaborated modelling, capture the correct behaviour of the problem of Gaussian rough surfaces with self-affine spectrum. The two main criticisms are:

1) that linearity in area-load occurs only at asymptotically low loads in BGT models, and otherwise a large spurious dependence on breadth parameter 
$\alpha$ (as defined by Nayak, 1971) is predicted. In contrast, Persson's theory is not apparently affected by $\alpha$ and shows a linear behavior between contact area and load up to $10-15 \%$ of the nominal contact area.

2 ) that the load-separation relationship should also be qualitatively different, and even in the asymptotic range of high separation (Persson, 2007).

We illustrate these two deviations with respect to the simplest $\mathrm{GW}-\mathrm{McCool}$ model (McCool, 1992) which obtains area-separation and force-separation as follows

$$
\begin{aligned}
\frac{A_{c}(t)}{A_{0}} & =\frac{\pi \sigma_{s} R D_{\text {sum }}}{\sqrt{2 \pi}} I_{1}^{g}(t) \\
\frac{F(t)}{A_{0}} & =\frac{4}{3 \sqrt{2 \pi}} E^{*}\left(R \sigma^{3}\right)^{1 / 2} D_{\text {sum }} I_{3 / 2}^{g}(t)
\end{aligned}
$$

where $I_{n}^{g}(t)=\int_{t}^{\infty} d \xi(\xi-t)^{n} \exp \left(-\xi^{2} / 2\right)$, and $\sigma$ is rms amplitude of summit heights, $t=s / \sigma$ is dimensionless separation, $R$ their radii, $D_{\text {sum }}$ the density of summits, and $E^{*}$ the plain strain elastic modulus of the contacting materials. From random process theory (Nayak, 1971), we can take

$$
D_{\text {sum }}=\frac{1}{6 \pi \sqrt{3}} \frac{m_{4}}{m_{2}} \quad ; \quad \frac{1}{R}=\frac{8}{3} \sqrt{\frac{m_{4}}{\pi}} \quad ; \quad \sigma=\sqrt{m_{0}}\left(1-\frac{0.9}{\alpha}\right)
$$

and hence $R \sigma D_{\text {sum }}=\frac{1}{48} \sqrt{\frac{3}{\pi}(\alpha-0.9)}$, where $m_{0}, m_{2}, m_{4}$ are the moments of the PSD, or else the variance of surface heights, slopes and curvatures.

Further, the slope in the area-load relationship is found easily to be

$$
\frac{A_{c}(t) \Omega}{F(t)}=\frac{3^{3 / 2} \pi}{8 \sqrt{2}}\left(\sqrt{\frac{1}{\alpha}}\right)^{1 / 2} \frac{I_{1}^{g}(t)}{I_{3 / 2}^{g}(t)} \simeq \frac{3^{3 / 2} \pi}{8 \sqrt{2}}\left(\sqrt{\frac{1}{\alpha}}\right)^{1 / 2}(1+0.148 t)
$$

where $\Omega=E^{*} \sqrt{m_{2} / \pi}$, showing dependence on bandwidth. This trend is in agreement with all other asperity contact theories at finite separations, disregarding the fact that at very large separations, the more refined ones predict linearity (Carbone \& Bottiglione, 2008). However, the change with $\alpha^{-1 / 4}$ is largely erroneous. It is clear that this factor, being possibly very large for very low fractal dimensions, may result in a slope change of a factor $1000^{-1 / 4}=$ 0.178 , i.e. almost an order of magnitude in the quite extreme cases.

Regarding the force-separation law (and its derivative, the stiffness), dividing this for the load itself, gives for the GW-McCool model (notice that we omit the dependence on the rms amplitude $\sigma$ as we differentiate with respect to $t$ )

$$
-\frac{\partial}{\partial t} \frac{F(t)}{A_{0}} / \frac{F(t)}{A_{0}}=-I_{3 / 2}^{g \prime}(t) / I_{3 / 2}^{g}(t) \simeq 0.92(1+t)
$$

giving for reasonable dimensionless separations $t=1,3$ a value between 2 and 3.5 . 
BGT models do not change this result significantly, as the load-separation is not affected, contrary to the area-load, significantly by bandwidth, so that we can take the asymptotic expression at high separations $\frac{F(t)}{A_{0}} \sim t^{-1} \exp \left(-\frac{t^{2}}{2}\right)$, which differentiated gives

$$
-\frac{\partial}{\partial t} \frac{F(t)}{A_{0}} / \frac{F(t)}{A_{0}} \sim \frac{\left(t^{-2}+1\right) \exp \left(-\frac{t^{2}}{2}\right)}{t^{-1} \exp \left(-\frac{t^{2}}{2}\right)} \sim t
$$

giving again for reasonable dimensionless separations $t=1,3$ a value between 1 and 3. Obviously one can fit a single value for the slope, finding an average value.

Persson (2007) derives from elastic energy concepts and with extensive derivations and correcting factors, a force-separation law $\frac{F(t)}{A_{0}} \sim \exp \left(-\frac{t}{\beta}\right)$, where $\beta$ is a corrective factor of the order $0.5-1$ (higher values being for low fractal dimensions), which however does not depend on separation and hence is a fixed value for a given system. This results in

$$
-\frac{\partial}{\partial t} \frac{F(t)}{A_{0}} / \frac{F(t)}{A_{0}} \sim \frac{1}{\beta}
$$

Therefore Persson's $\beta=0.5-1$ corresponds to a slope between 1 and 2 which however does not depend on separation this time, but only on the spectrum geometrical characteristics. However, it is hard to say that results are qualitatively different. Proportionality of stiffness with load is approximately true for both theories, and perhaps more rigorously for Persson's theory - but this hasn't been shown to be so extensively valid in experimental surfaces, with few good exceptions (Lorenz et al., 2010). Numerical simulations of the dependence of stiffness on the applied squeezing pressure show that the dependence on the large wavelengths component of roughness induces large scatter in results and finite-size effects which induce sublinear power-law scaling (Pastewka et al, 2013) which means higher values for $\beta$ also depending on separation, but this is important in the more general context of the reliability of a Gaussian random process approximation.

We shall return to the very basic point: are real surface Gaussian? Clearly, it did not seem so at the time of the original Greenwood and Williamson (1966) paper, if one looks at Fig.6 where a mild steel had been abraded and then put to slide against copper: so we are unlikely to make a good approximation with a Gaussian if surfaces have undergone wear. We can estimate what happens in wear processes from the closely related problem of Chemical Mechanical Polishing in planarization of integrated circuits (Vasilev et al, 2013, Borucki, 2002, Borucki et al. 2004). Indeed, polishing causes high asperities wearing faster than low asperities, and Borucki derived, based on Archard law for wear and the GW model, an equation similar to a differential Hamilton-Jacobi equation for the distribution of asperity heights. High peaks in the tail of the distribution appear, the tail becomes increasingly worn out and indeed even a delta function 
can result. Stein et al (1996) show indeed experimental distribution of heights which resemble inverted Weibull, i.e. where the tail is on the lower end of the height distribution, and the right tail has been completely removed.

Nayak (1971) is the classical reference for random process theory, yet he himself writes that "it is clear that many surfaces are non-Gaussian; but it is equally clear that many surface are Gaussian". Central Limit Theorem (CLT) is a weak justification for Gaussian height distribution, and even numerical realization intended to be Gaussian in principle, sometimes fail to do so. In fact, the longest wavelengths will dominate the height distribution, and there are generally only very few of them. More in general, Persson et al (2005) has a number of surface height distributions also from experiments, but it is unclear if they are more or less Gaussian, and no statistical test is conducted.

Here, we shall relax the assumption of Gaussian height distribution, while maintaining the assumption of asperity behaviour, and in particular, we shall consider the extreme case of a Weibull distribution of asperities approaching a rigid plane from the bounded side of the distribution. It will be shown that this leads to dramatic changes in the results, much more significant than the differences between asperity models and Persson's theory, which have been found in the specific, although important, case of Gaussian surfaces.

\section{$2 \quad$ Weibull asperity distribution}

According to the standard GW integration process as a function of asperity height, we obtain number of asperities in contact, area and load, integrating

$$
\begin{aligned}
& n=N \frac{a}{\sigma_{s}} \int_{d_{0}}^{\infty}\left(\frac{z_{s}}{\sigma_{s}}\right)^{a-1} \exp \left(-\left(\frac{z_{s}}{\sigma_{s}}\right)^{a}\right) d z_{s} \\
& A=N \pi R \frac{a}{\sigma_{s}} \int_{d_{0}}^{\infty}\left(z_{s}-d_{0}\right)\left(\frac{z_{s}}{\sigma_{s}}\right)^{a-1} \exp \left(-\left(\frac{z_{s}}{\sigma_{s}}\right)^{a}\right) d z_{s} \\
& P=N \frac{a}{\sigma_{s}} \int_{d_{0}}^{\infty} \frac{4}{3} \frac{E}{R}(R d)^{3 / 2}\left(\frac{z_{s}}{\sigma_{s}}\right)^{a-1} \exp \left(-\left(\frac{z_{s}}{\sigma_{s}}\right)^{a}\right) d z_{s}
\end{aligned}
$$

where $N$ is number of asperities, $\sigma_{s}$ the amplitude parameter (which does not correspond to the $\sigma$ introduced above as rms amplitude of summit heights distribution), $R$ the radius of asperities.

Then, changing variable to $d+d_{0}=z_{s}$, normalizing the heights by $\sigma_{s}$, the lower extreme of integration is 0 and defining

$$
I_{n}\left(d_{0}^{*}\right)=\int_{0}^{\infty}\left(d^{*}\right)^{n}\left(d^{*}+d_{0}^{*}\right)^{a-1} \exp \left(-\left(d^{*}+d_{0}^{*}\right)^{a}\right) d d^{*}
$$


we obtain

$$
\begin{aligned}
n\left(d_{0}^{*}\right) & =N a I_{0}\left(d_{0}^{*}\right) \\
A\left(d_{0}^{*}\right) & =\left(N R \sigma_{s}\right) \pi a I_{1}\left(d_{0}^{*}\right) \\
P\left(d_{0}^{*}\right) & =\frac{4}{3} N a E R^{1 / 2} \sigma_{s}^{3 / 2} I_{3 / 2}\left(d_{0}^{*}\right)
\end{aligned}
$$

and so the ratio between stiffness and load at a given separation is

$$
\frac{\partial}{\partial d_{0}^{*}} P\left(d_{0}^{*}\right) / P\left(d_{0}^{*}\right)=I_{3 / 2}^{\prime}\left(d_{0}^{*}\right) / I_{3 / 2}\left(d_{0}^{*}\right)
$$

We write non dimensional relationships,

$$
\begin{aligned}
& \frac{A\left(d_{0}^{*}\right)}{A_{0}^{g}}=\frac{a \sqrt{2 \pi}}{\sqrt{\Gamma\left(1+\frac{2}{a}\right)-\Gamma\left(1+\frac{1}{a}\right)^{2}}} I_{1}\left(d_{0}^{*}\right) \\
& \frac{P\left(d_{0}^{*}\right)}{P_{0}^{g}}=\frac{a \sqrt{2 \pi}}{\left(\Gamma\left(1+\frac{2}{a}\right)-\Gamma\left(1+\frac{1}{a}\right)^{2}\right)^{3 / 4}} I_{3 / 2}\left(d_{0}^{*}\right)
\end{aligned}
$$

Here, we have used the rms amplitude for a Weibull height distribution $\left.h_{r m s}=\sigma_{s} \sqrt{\Gamma\left(1+\frac{2}{a}\right)-\Gamma\left(1+\frac{1}{a}\right)^{2}}\right)$, and we have defined the factors $P_{0}^{g}=$ $\frac{4}{3} \frac{N}{\sqrt{2 \pi}} E R^{1 / 2} h_{r m s}^{3 / 2}$ and $A_{0}^{g}=\sqrt{\pi / 2}\left(N R h_{r m s}\right)$ to make easier comparison with the Gauss distribution result. In the Gaussian case, we return essentially to the McCool model, although here we are normalizing by the $P_{0}^{g}, A_{0}^{g}$ factors..

\section{Results}

In Fig.1 we plot the standard results for the asperity model with Weibull distribution, where it is clear that area-load is almost linear and depends very little from the Weibull shape parameter. The bandwidth dependence cannot appear from this plot, but it is not relevant. On the other hand, the loadseparation plots (Fig.1b) show very large change of the trend, and notice that the Persson's result is intermediate between the exponential distribution and the Rayleigh distribution ( $a=1,2$ respectively). So nothing really new here.

More interesting is the case when the contact occurs on the tail of the distribution, as in Fig.2. Here, area-load continues to be mostly unaffected by the Weibull parameter, although it becomes more non-linear, but on the contrary the load-separation becomes extremely non-linear even in the log-linear scale. Clearly, this is mechanically simple to explain, as we have a large number of asperities near the point of initial contact, so stiffness increases very rapidly upon further indentation, and then tends to a very "soft" value. 

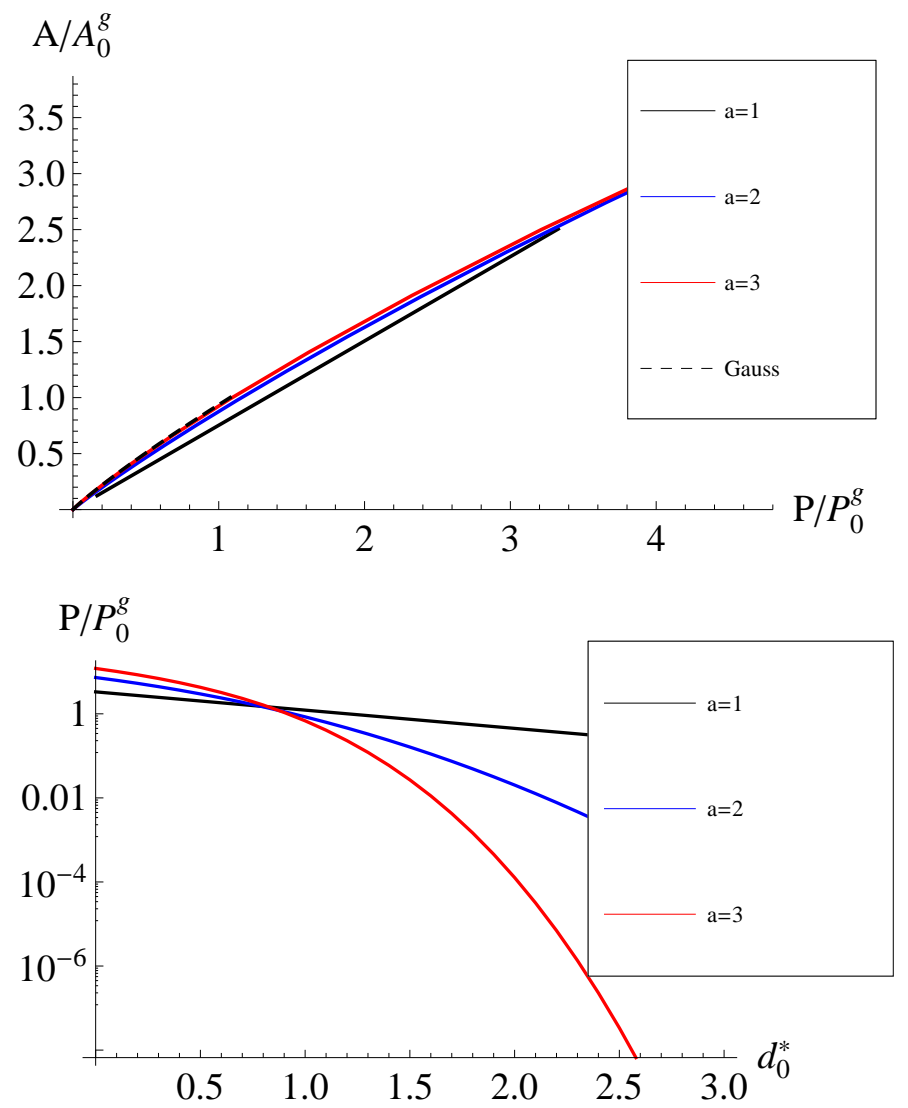

(a)

Fig.1. Area-load (a), and load-separation, for the approach on the tail side of the Weibull tail. Notice that separation is positive here as in standard asperity models. 

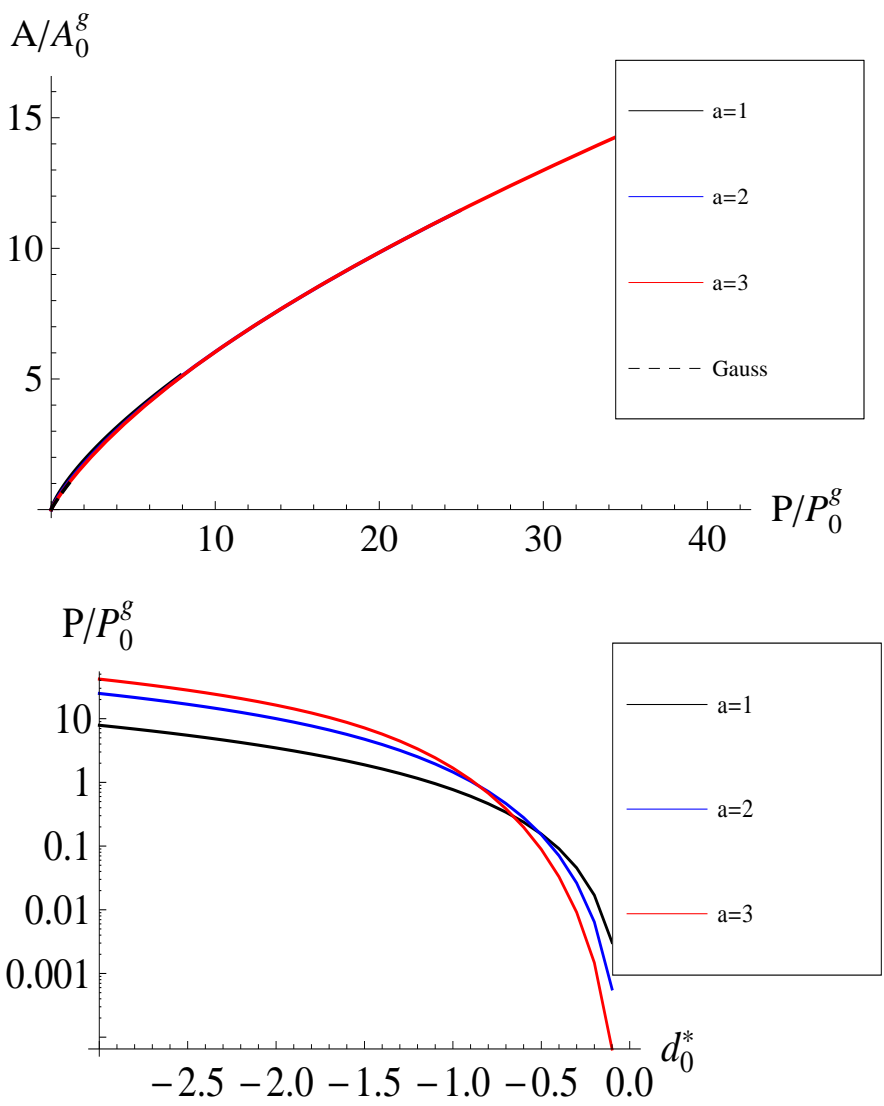

(a)

Fig.2. Area-load (a), and load-separation, for the approach on the bounded side of the Weibull tail. Notice that separation is negative in this case as zero corresponds to just zero contact.

We further derive the results for stiffness in Fig.3, showing in Fig.3a the results for the standard asperity model, showing the ratio stiffness to load is actually lower than the Gaussian one (and hence quite similar to the Persson's one), for low Weibull parameter, as observed already when looking at the loadseparation plots. Viceversa, when approach is on the bounded side of the tail, the stiffness/load ratio approaches the value 1 only at very large indentation, and otherwise it can increase substantially, being in principle infinite at the point of initial contact. This suggests that the detail of the height distribution matter, when considering stiffness. 


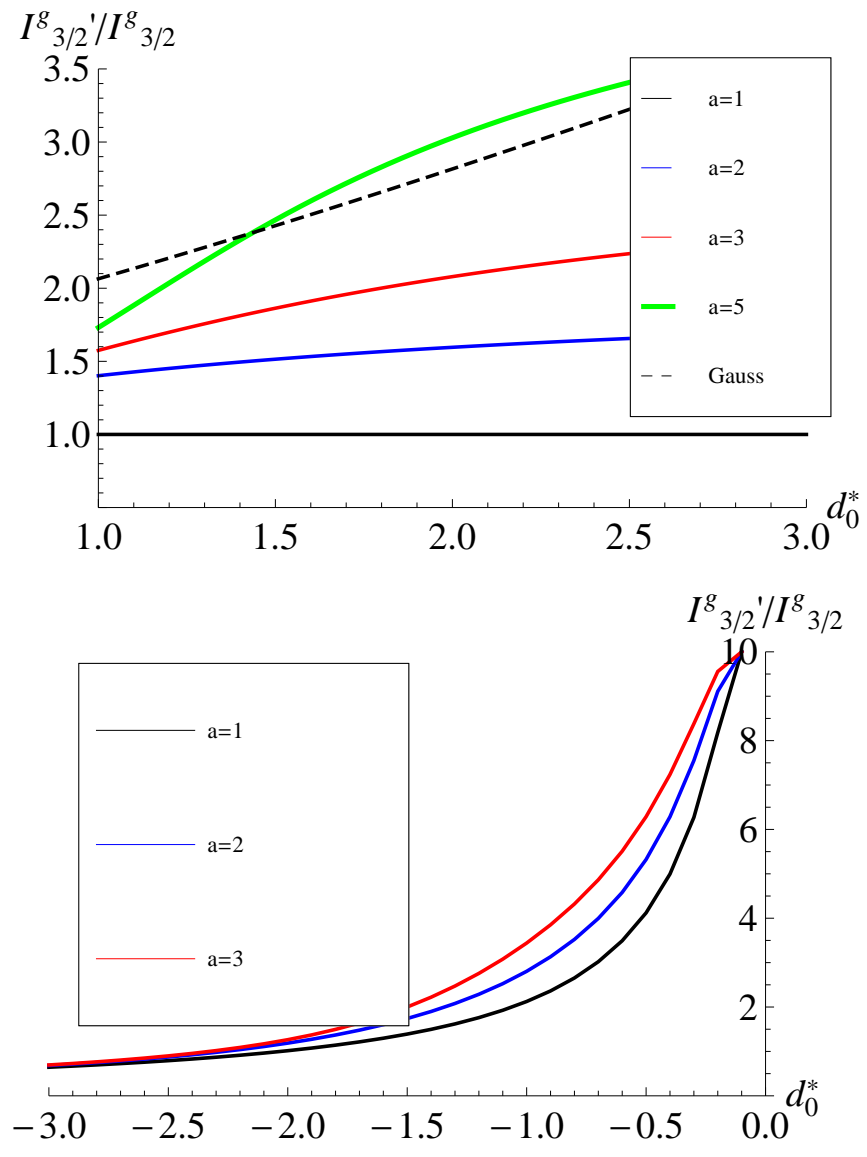

(a)

(b)

Fig.3. Stiffness-load ratio, for the standard approach on the tail side of the Weibull (a), or on the bounded side (b)

\section{Conclusion}

We have shown that, while recent theories of contact mechanics like that of Persson, have certainly improved our understanding of the Gaussian surface problem, the assumption itself is quite strong, especially when it comes to stiffness and relation load-separation, which largely depend on the asperity height distribution.

\section{$5 \quad$ References}

Borucki, L. Mathematical modeling of polish rate decay in chemical-mechanical polishing. J. Engng. Math. 43 (2002) 105-114 
Borucki, L. J., Witelski, T., Please, C., Kramer, P. R., \& Schwendeman, D. (2004). A theory of pad conditioning for chemical-mechanical polishing. Journal of engineering mathematics, 50(1), 1-24.

Carbone, G., \& Bottiglione, F. (2008). Asperity contact theories: Do they predict linearity between contact area and load?. Journal of the Mechanics and Physics of Solids, 56(8), 2555-2572.

Greenwood, J.A., Williamson, J.B.P., 1966. Contact of nominally flat surfaces. Proc. R. Soc. London A295, 300-319.

Lorenz, B., Carbone, G., \& Schulze, C. (2010). Average separation between a rough surface and a rubber block: Comparison between theories and experiments. Wear, 268(7), 984-990.

McCool, J. I. (1992). Non-Gaussian effects in microcontact. International Journal of Machine Tools and Manufacture, 32(1), 115-123.

Nayak, P. R. 1971. Random process model of rough surfaces. Journal of Tribology, 93(3), 398-407.

Pastewka, L., Prodanov, N., Lorenz, B., Müser, M. H., Robbins, M. O., \& Persson, B. N. (2013). Finite-size scaling in the interfacial stiffness of rough elastic contacts. Physical Review E, 87(6), 062809.

Persson, B. N. 2001. Theory of rubber friction and contact mechanics. The Journal of Chemical Physics, 115(8), 3840-3861.

Persson, B N J, Albohr, O, Tartaglino, U, Volokitin A I and Tosatti. E 2005. On the nature of surface roughness with application to contact mechanics, sealing, rubber friction and adhesion J. Phys.: Condens. Matter 17 R1, http://arxiv.org/pdf/cond-mat/0502419.pdf)

Persson, B. N. J. (2007). Relation between interfacial separation and load: a general theory of contact mechanics. Physical review letters, 99(12), 125502.

Stein, D., Hetherington, D., Dugger, M., \& Stout, T. (1996). Optical interferometry for surface measurements of CMP pads. Journal of Electronic Materials, 25(10), 1623-1627.

Vasilev, B., Bott, S., Rzehak, R., \& Bartha, J. W. (2013). Pad roughness evolution during break-in and its abrasion due to the pad-wafer contact in oxide CMP. Microelectronic Engineering, 111, 21-28. 